\title{
Mortality in adolescent trauma: a comparison of children's, mixed and adult major trauma centres
}

\author{
Jordan Evans (1) , ${ }^{1}$ Hannah Murch, ${ }^{1}$ Roisin Begley, ${ }^{2}$ Damian Roland (ㄷ), \\ Mark D Lyttle, ${ }^{2}$ Omar Bouamra, ${ }^{5}$ Stephen Mullen (1) ${ }^{6}$
}

\begin{abstract}
Handling editor Caroline
Leech

${ }^{1}$ Paediatric Emergency Department, University Hospital of Wales, Cardiff, UK ${ }^{2}$ Emergency Department, Bristol Royal Children's Hospital, Bristol, UK

${ }^{3}$ Health Sciences, University of Leicester, Leicester, UK

${ }^{4}$ Paediatric Emergency Medicine Leicester Academic (PEMLA) Group, Leicester Hospitals, Leicester, UK

${ }^{5}$ The Trauma Audit and Research Network, Salford, UK ${ }^{6}$ Paediatric Emergency Department, Royal Belfast Hospital for Sick Children, Belfast, UK
\end{abstract}

Correspondence to Stephen Mullen, Paediatric Emergency Department, Royal Belfast Hospital for Sick Children, Belfast BT12 6BA, UK; smullen001@googlemail.com

Received 11 July 2020 Revised 21 February 2021 Accepted 22 February 2021 Published Online First 30 March 2021
ABSTRACT

Objective We aimed to compare adolescent mortality rates between different types of major trauma centre (MTC or level 1; adult, children's and mixed).

Methods Data were obtained from TARN (Trauma Audit Research Network) from English sites over a 6year period (2012-2018), with adolescence defined as 10-24.99 years. Results are presented using descriptive statistics. Patient characteristics were compared using the Kruskal-Wallis test with Dunn's post-hoc analysis for pairwise comparison and $\chi^{2}$ test for categorical variables. Results 21033 cases met inclusion criteria. Traumarelated 30-day crude mortality rates by MTC type were 2.5\% (children's), 4.4\% (mixed) and 4.9\% (adult). Logistic regression accounting for injury severity, mechanism of injury, physiological parameters and 'hospital ID', resulted in adjusted odds of mortality of $2.41(95 \% \mathrm{Cl} 1.31$ to $4.43 ; p=0.005)$ and $1.85(95 \%$ Cl 1.03 to 3.35; $p=0.041)$ in adult and mixed MTCs, respectively when compared with children's MTCs. In three subgroup analyses the same trend was noted. In adolescents aged 14-17.99 years old, those managed in a children's MTC had the lowest mortality rate at $2.5 \%$, compared with $4.9 \%$ in adult MTCs and $4.4 \%$ in mixed MTCs (no statistical difference between children's and mixed). In cases of major trauma (Injury Severity Score $>15$ ) the adjusted odds of mortality were also greater in the mixed and adult MTC groups when compared with the children's MTC. Median length of stay (LOS) and intensive care unit LoS were comparable for all MTC types. Patients managed in children's MTCs were less likely to have a CT scan ( $46.2 \%$ vs $62.8 \%$ mixed vs $64 \%$ adult).

Conclusions Children's MTC have lower crude and adjusted 30-day mortality rates for adolescent trauma. Further research is required in this field to identify the factors that may have influenced these findings.

\section{SLinked}

- http://dx.doi.org/10.1136/ emermed-2020-211105

Check for updates

(C) Author(s) (or their employer(s)) 2021. No commercial re-use. See rights and permissions. Published by BMJ.

To cite: Evans J, Murch $\mathrm{H}$, Begley $\mathrm{R}$, et al. Emerg Med J 2021:38:488-494.

\section{INTRODUCTION}

In the UK, trauma accounts for 6 million ED attendances, 720000 admissions and 17000 deaths a year. ${ }^{1}$ Worldwide, approximately 5.8 million people die each year as a result of trauma, accounting for $10 \%$ of the world's deaths. ${ }^{2}$ Trauma is the leading cause of death and acquired disability in adolescents, with road traffic collision (RTC) the most common mechanism. ${ }^{3}$ Regional major trauma networks, established in England in 2012 consist of major trauma centres (MTC; equivalent to level 1 trauma centres) and linked trauma units (TU; equivalent to level $2 / 3$ trauma centres). In the current system configuration, the 27 MTCs, which receive

\section{Key messages}

What is already known on this subject

- Trauma is a leading cause of mortality and morbidity in adolescence.

- Adolescent trauma patients may be managed in either a children's, mixed or adult trauma centre.

- The current evidence base on outcomes by centre type is inconsistent. Some report a higher mortality in adolescent trauma patients managed in an adult and mixed major trauma centres (MTCs) when compared with children's, while others have reported no difference.

\section{What this study adds}

- Trauma Audit Research Network (TARN) data from English MTCs demonstrates lower crude and adjusted mortality rates for adolescent trauma patients attending a children's MTC.

- This association persists in cases of severe trauma (Injury Severity Score $>15$ ).

- There was no difference in length of stay between MTC types (children's, mixed or adult), although variation existed in $\mathrm{CT}$ rates and time from arrival to $\mathrm{CT}$.

the most seriously injured patients, are classified as adult ( $\mathrm{n}=11$; patients aged $\geq 16$ years), children's $(n=5$; patients $<16$ years) or mixed $(n=11)$.

The WHO and UNICEF define adolescence as the age range 10-19 years, with 'young people' classified as those aged 10-24 years. ${ }^{4} 5$ Recent recommendations on the age range for adolescence state this should include those aged 10-24 years to better acknowledge 'recognised shifts in events of biological growth and social role transitions', a stance supported in the UK by the $\mathrm{RCPCH}^{7}$ In the USA, youth violence prevention strategies are targeted to the 10-24 years age bracket. ${ }^{8}$

Adolescents are a unique cohort in that, dependent on age, they may be managed in either paediatric, adult or mixed trauma services. In England, ambulance services triage and convey patients under 16 years to a children's MTC and those over 16 years to an adult MTC. A mixed MTC accepts patients of all ages. In rare circumstances, the age of an unconscious adolescent may be unclear and the patient may be conveyed to the incorrect trauma centre for their age by the ambulance service. While the principles of trauma management overlap between paediatric and adult guidelines, there exist 
subtle but important differences, including imaging strategies, the role of permissive hypotension and conservative management of abdominal organ injuries. ${ }^{9} 10$

It is not known whether these differing strategies impact outcomes in this cohort, or in what age groups differences may exist. There are no national published data comparing outcomes in this configuration for adolescent trauma between MTC types, and there are limited international studies. The majority come from the USA, a comparable trauma system to the UK, but findings are inconsistent. Some report higher mortality in adult and mixed MTCs compared with children's MTCs, while others report no association. ${ }^{11-13}$

The primary aim of this study was to identify mortality rates for severely injured adolescents in England in different MTC types. The secondary aims were to describe length of stay (LoS), number of critical care days (intensive care unit (ICU) LoS), time to CT and the most senior clinician present at the initial resuscitation in MTC types.

\section{METHODS}

\section{Study design and setting}

In this cross-sectional study we analysed data collected in the national Trauma Audit Research Network (TARN) database over a 6 years and 9 months period (1 April 2012 to
31 December 2018), with the commencement date selected to coincide with the implementation of trauma networks in England. ${ }^{14}$ The study was limited to English sites as there were no MTCs in Wales or Northern Ireland during the study period, and Scottish trauma data are captured elsewhere (Scottish Trauma Audit Group).

\section{Study population}

The TARN database includes patients of any age who sustain injury resulting in hospital admission for 3 days or greater, critical care admission, transfer to a tertiary/specialist centre or in-hospital death within 30 days. Injuries are assigned an Abbreviated Injury Scale (AIS) score, ranging from 1 (minor injury) to 6 (an injury that is thought to be 'incompatible with life'). ${ }^{15}$ The Injury Severity Score (ISS), derived by adding the squares of the three highest scoring body regions, ranges from 1 to 75 , with major trauma defined as ISS $>15 .{ }^{14}$ Data are entered into the TARN database by local coordinators, using retrospective chart review methodology.

Datasets were included for all adolescent trauma episodes recorded in TARN with initial presentations to any MTC. Adolescence was defined as 10-24.99 years in line with international guidance. ${ }^{216}$ Prehospital deaths are not recorded by TARN. Cases that initially presented to TUs were excluded

Figure 1. Total number of adolescent trauma cases coded by Trauma Audit Research Network

(TARN) between 1 April 2012 to 31 December 2018. Subcategorised by type of major trauma centre (MTC) (children's, mixed, adult).

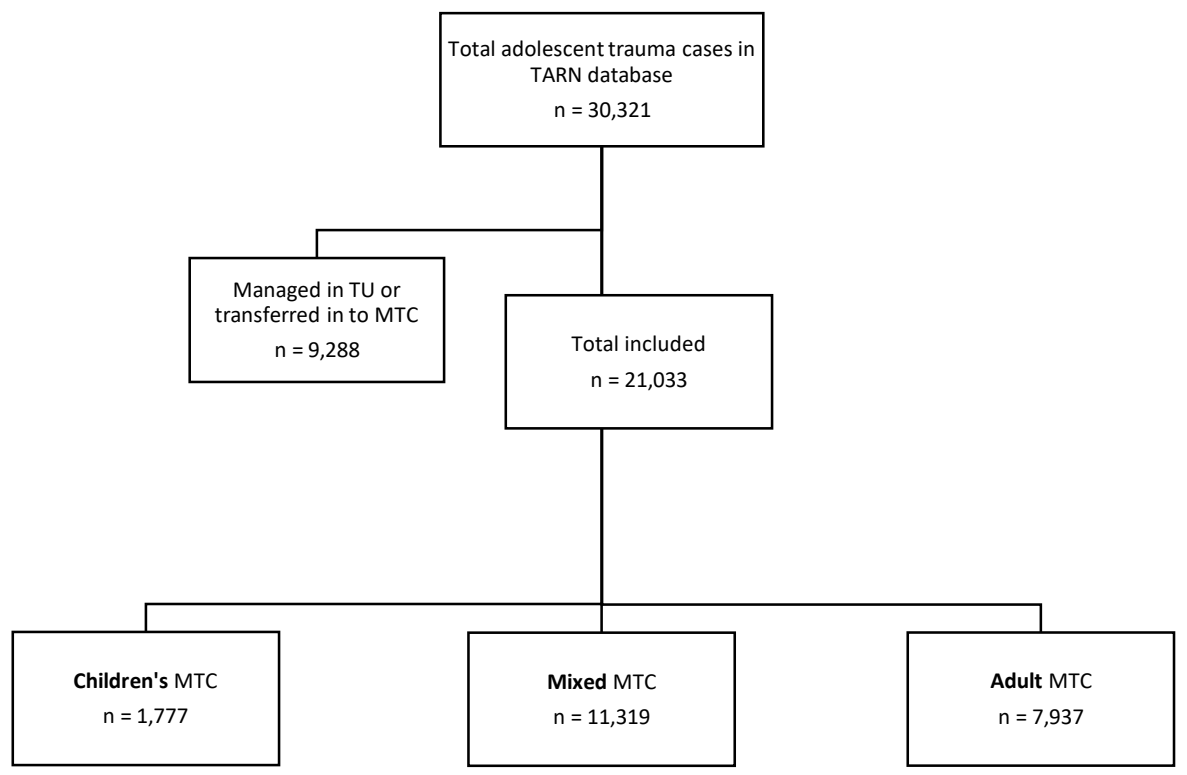

Figure 1 Total number of adolescent trauma cases coded by Trauma Audit Research Network (TARN) between 1 April 2012 to 31 December 2018. Subcategorised by type of major trauma centre (MTC) (children's, mixed, adult). 
to reduce confounding from wider heterogeneity in initial management.

\section{Outcomes}

The primary outcome measure was mortality within 30 days of traumatic event, with comparison between children's, adult and mixed MTCs. Secondary outcomes selected as measures of morbidity included length of stay and number of intensive care bed days. Intercentre comparison was undertaken for seniority of clinician managing initial management, and time taken for CT scanning where relevant.

\section{Statistical analysis}

In this analysis, the null hypothesis was that there was no difference in mortality rates between MTC types. Patient characteristics, when continuous, were compared using the Kruskal-Wallis test with Dunn's post-hoc analysis for pairwise comparison and $\chi^{2}$ test for categorical variables. To allow for variation between sites, a logistic mixed effect (random intercept) regression was used. The logistic regression adjusted for mechanism of injury, GCS, ISS, systolic blood pressure (SBP), pulse rate, comorbidities and site institution with the inclusion of 'hospital ID'. This is necessary to take into account the clustering of patients within each hospital to enable analysis of not only unexplained variance between patients but also between hospitals. Hosmer and Lemeshow (H-L) was used to test for goodness-of-fit and the $\mathrm{C}$ statistic by means of the area under the receiver operating curve (AROC) to assess the discriminating power of the statistical model. As TARN data are inputted retrospectively, required fields may not be recorded in the clinical notes. Missing data from the covariates were imputed using multiple imputation under the assumption of missingness at random mechanism and applying Rubin's rule on the 10 imputed sets. The same statistical analysis was undertaken for all subanalyses. All the analyses were performed using Stata V.14 software (StataCorp).

We subcategorised the study cohort for three planned subanalyses for which we assessed crude and adjusted mortality comparing outcomes based on MTC type (children's, mixed and adult). In the first subgroup analysis, the cohort was split into two age ranges, $10-15.99$ years and $16-24.99$ years. In assessing trauma outcomes for the breadth of adolescences, from 10 to 24 years, concerns may be expressed about the validity of comparing trauma in a 10 -year-old to a 24 -year-old due to potential differences in physiology and mechanism of injury. In restricting the age range, a more homogenous population may be assessed. These age boundaries were selected to account for the cut-off age of 16 years for children's versus adult MTCs. The second subanalysis focused on all adolescent trauma patients within the age group 14-17.99 years. This age range was selected pragmatically by consensus of the study team, to represent upper and lower boundaries that paediatric and adult services, respectively may feel comfortable accepting, and reflect reasonable presumed age deviations for those making decisions prehospital. A further planned subanalysis assessed patients specifically with major trauma (ISS >15) for the full cohort (age 10 years-24 years) and those aged 14-17.99 years. The same statistical analyses were performed on these subgroups to adjust for potential confounders.

\section{Patient and public involvement}

Patients or the public were not involved in the design, conduct, reporting or dissemination plans of this study.

\section{RESULTS}

\section{Demographic and injury pattern data}

A total of 30321 adolescent trauma cases were identified of which 21033 fulfilled eligibility criteria for this analysis. Of these, 11319 (54\%) presented to a mixed MTC, 7937 (38\%) to an adult MTC and 1777 (8\%) to a children's MTC (figure 1). $2569(12.2 \%)$ were aged $10-13.99$ years, $4471(21.3 \%)$ aged 14-17.99 years and 13993 (66.5\%) aged 18-24.99 years. Patients were predominantly male $(77.9 \%)$ (table 1$)$.

The initial physiological parameters were similar between groups but did demonstrate statistically significant differences between MTC cohorts. These differences were unlikely to be clinically significant (table 1). Overall, across all three types of MTC, the most common comorbidities were asthma, mental health conditions, alcohol/drug abuse and epilepsy. In $80 \%$ (16 883) of the total cohort, the patient had no comorbidities. Patients presenting to children's MTCs had fewer comorbidities.

The most common mechanism of injury was RTC $(52.6 \%$, $\mathrm{n}=11058$ ), with falls of any height accounting for $22.2 \%$ (4657). Falls $<2 \mathrm{~m}$ height accounted for a larger proportion of children's MTC cases $(20.5 \%)$ compared with mixed (10\%) and adult (9.5\%) MTCs. Stabbings and shootings were less common in children's MTCs (2.8\%) and highest in mixed MTCs (14.5\%) (table 2). Patients managed in children's MTCs had a median ISS of 10, whereas those managed in mixed or adult MTCs had a median ISS of 14 and 16 , respectively $(\mathrm{p}<0.001)$ (table 1 ). Anatomical regions with significant differences in median highest AIS between MTC types were the face, abdomen, pelvis and limbs (table 2).

\section{Seniority leading resuscitation, LoS and time to CT}

In most cases a consultant was present as the most senior clinician (table 1). Physicians in their first 4 years of training were the most senior clinician present in only a small proportion $(<5 \%)$ of cases, but this was most common in adult MTCs. Median LoS and ICU LoS were comparable for all MTC types although differences met statistical significance (table 1).

Patients managed in children's MTCs were less likely to have a CT scan, though the time taken to perform a CT was significantly longer in children's MTCs than other MTC types (table 1).

\section{Mortality}

Overall trauma-related crude 30-day mortality was 4.4\%, with significant differences in mortality rates between MTC types (children's 2.5\%; mixed 4.4\%; adult 4.9\%; $\mathrm{p}<0.0001$; table 1). When adjustments were made using logistic regression accounting for ISS, MOI, GCS, SBP, pulse rate, comorbidity, pupil reactivity and the random effect 'hospital ID', odds of mortality compared with children's MTCs were 2.41 (95\% CI 1.31 to $4.43 ; \mathrm{p}=0.005)$ and 1.85 (95\% CI 1.03 to 3.35 ; $\mathrm{p}=0.041$ ) in adult and mixed MTCs, respectively (table 3 ). The model showed a good C statistic (AROC) of 0.980 (95\% CI 0.977 to 0.984 ) and the goodness of fit showed a non-significant $\mathrm{H}-\mathrm{L}$ test $(\mathrm{p}=0.55)$, demonstrating that the model fits the data well.

\section{Subgroup analysis: $10-15.99$ years}

In the subgroup analysis of the 10-15.99 years old, those managed in a children's MTC had the lowest mortality rate at $2.3 \%$, compared with $3.5 \%$ in adult MTCs and $3.8 \%$ in mixed MTCs. In the adjusted analysis, the OR for mortality was higher in the mixed and adult MTCs in comparison to children's MTCs (statistically significant for mixed only) (table 3). 
Table 1 Demographic and outcome data for adolescent trauma cases coded by Trauma Audit Research Network

\begin{tabular}{|c|c|c|c|c|c|}
\hline & Children's MTC & Mixed MTC & Adult MTC & Total & $P$ value \\
\hline Total number (\%) & $1777(8.4 \%)$ & $11319(53.8 \%)$ & $7937(37.7 \%)$ & 21033 & \\
\hline $\begin{array}{l}\text { Age in years } \\
\text { Median (IQR) }\end{array}$ & $13.3(11.8-14.8)$ & $19.8(16.9-22.3)$ & $20.7(18.8-22.8)$ & $19.8(16.7-22.3)$ & $<0.0001$ \\
\hline Male n (\%) & $1260(70.9)$ & $8885(78.5)$ & $6247(78.7)$ & $16392(77.9)$ & $<0.0001$ \\
\hline Female $n(\%)$ & $517(29.1)$ & $2434(21.5)$ & $1690(21.3)$ & $4641(22.1)$ & \\
\hline \multicolumn{6}{|c|}{ Physiological parameters at presentation Median (IQR) } \\
\hline $\mathrm{GCS}^{*}$ & $15(14-15)$ & $15(14-15)$ & $15(14-15)$ & $15(14-15)$ & 0.999 \\
\hline Pulse & $90(79-106)$ & $88(75-102)$ & $88(75-103)$ & $88(75-103)$ & $<0.0001$ \\
\hline SBP $\mathrm{mm} \mathrm{Hg}$ & $118(109-127)$ & $126(114-139)$ & $129(116-142)$ & $126(114-139)$ & $<0.0001$ \\
\hline \multicolumn{6}{|c|}{ Comorbidity (Charlson Index) n (\%) } \\
\hline 0 & $1572(88.5)$ & 8929 (78.9) & $6382(80.4)$ & $16883(80.3)$ & $<0.0001$ \\
\hline $1-5$ & $113(6.4)$ & $1233(10.9)$ & $1024(12.9)$ & $2370(11.3)$ & \\
\hline $6-10$ & $2(0.1)$ & $26(0.2)$ & $14(0.2)$ & $42(0.2)$ & \\
\hline$>10$ & $1(0.06)$ & $3(0.03)$ & $3(0.04)$ & $7(0.03)$ & \\
\hline \multicolumn{6}{|c|}{ Most senior clinician n (\%) } \\
\hline Consultant & $1207(67.9)$ & $9214(81.4)$ & $5858(73.8)$ & $16279(77.4)$ & $<0.0001$ \\
\hline Associate Spec. & $24(1.4)$ & $51(0.5)$ & $140(1.8)$ & $215(1.0)$ & \\
\hline ST3+ & $238(13.4)$ & $535(4.7)$ & $655(8.3)$ & $1428(6.8)$ & \\
\hline FP/ST 1-2 & $48(2.7)$ & $295(2.6)$ & $343(4.3)$ & $686(3.3)$ & \\
\hline Other & $260(14.6)$ & $1224(10.8)$ & $941(11.9)$ & $2425(11.5)$ & \\
\hline Mortality n (\%) & $44(2.5 \%)$ & $501(4.4 \%)$ & $387(4.9 \%)$ & $932(4.4 \%)$ & $<0.0001$ \\
\hline \multicolumn{6}{|l|}{ Length of stay } \\
\hline $\begin{array}{l}\text { LoS in days } \\
\text { Median (IQR) }\end{array}$ & $6(4-11)$ & $6(4-12)$ & $7(4-12)$ & $6(4-12)$ & 0.0003 \\
\hline $\begin{array}{l}\text { ICU LoS in days } \\
\text { Median (IQR) }\end{array}$ & $2(1-5)$ & $2(1-6)$ & $3(1-8)$ & $2(1-6)$ & $<0.0001$ \\
\hline \multicolumn{6}{|l|}{ CT } \\
\hline CT performed n (\%) & $821(46.2)$ & $7105(62.8)$ & $5080(64)$ & $13006(61.8)$ & $<0.0001$ \\
\hline $\begin{array}{l}\text { Time to CT min } \\
\text { Median (IQR) }\end{array}$ & $40(26-55)$ & $28(19-44)$ & $23(14-36)$ & $27(17-42)$ & $<0.0001$ \\
\hline
\end{tabular}

Subcategorised by MTC type.

${ }^{*}$ Denotes no statistical difference between subcategorisation of MTC (only applicable to GCS).

FP, foundation programme trainee; ICU, intensive care unit; LoS, length of stay; MTC, major trauma centre; SBP, systolic blood pressure; ST, specialty trainee.

\section{Subgroup analysis: $16-24.99$ years}

In the subgroup analysis of the 16-24.99 years old cohort, there was significant variation in participants number between MTC's (children's MTC $\mathrm{n}=17$, mixed MTC $\mathrm{n}=9153$ and adult MTC $n=7507$; table 4). Those managed in a children's MTC had the highest mortality rate at $17.7 \%$, compared with $5 \%$ in adult MTCs and $4.6 \%$ in mixed MTCs. In the adjusted analysis, the OR for mortality was higher in adult and mixed MTC's compared with children's MTCs, but this did not meet statistical significance (table 3).

\section{Subgroup analysis: $14-17.99$ years}

In the subgroup analysis of the 14-17.99 years old, those managed in a children's MTC had the lowest mortality rate at $2.5 \%$, compared with $4.9 \%$ in adult MTCs and $4.4 \%$ in mixed MTCs. In the adjusted analysis, the OR for mortality was significantly higher in adult MTCs in comparison to children's MTCs. There was no statistically significant difference between mixed MTCs in comparison to children's MTCs (table 3).

\section{Subgroup analysis: ISS >15 (major trauma) in adolescents (10-24.99 years)}

Table 5 displays the 30-day crude and adjusted odds of mortality for major trauma (ISS >15). The adjusted odds of mortality are greater in the mixed and adult MTC groups when compared with the children's MTC. This association is replicated in the 14-17.99 years subgroup for major trauma (table 5).

\section{DISCUSSION}

Using English trauma registry data, we have demonstrated lower 30-day crude mortality rates for adolescent trauma patients (10-24.99 years) attending children's MTCs in comparison to adult or mixed MTCs. This association persisted after adjustment for potential confounding factors, and in subgroup analyses comparing outcomes for those with major trauma (ISS >15) and those aged 14-17.99 years (with or without major trauma).

When the cohort was divided into young (10-15.99 years) and older adolescence (16-24.99 years), the trend for an adjusted improved mortality rate in those attending children's MTCs remained. In the young adolescent group (10-15.99 years), the adjusted odds of mortality were 2.2 times higher for those who attended a mixed MTC compared with a children's MTC, which was statically significant. In the comparison of adult MTCs to children's MTCs for the 10-15.99 years age group, the adjusted odds of mortality was 2.9 , although this was not statically significant.

For the older adolescent cohort (16-24.99 years) those managed in a children's MTC had the highest mortality rate at $17.7 \%$, compared with 5\% in adult MTCs and $4.6 \%$ in mixed MTCs. The adjusted odds of mortality however continue to 


\section{Original research}

Table 2 Mechanism of injury, ISS and AIS for adolescent patients coded by Trauma Audit Research Network subcategorised by MTC type

\begin{tabular}{|c|c|c|c|c|c|}
\hline & $\begin{array}{l}\text { Children's } \\
\text { MTC }\end{array}$ & $\begin{array}{l}\text { Mixed } \\
\text { MTC }\end{array}$ & $\begin{array}{l}\text { Adult } \\
\text { MTC }\end{array}$ & Total & $P$ value \\
\hline \multicolumn{6}{|c|}{ Mechanism of injury (MOI) n (\%) } \\
\hline RTC & $931(52.4)$ & $5809(51.3)$ & $4318(54.4)$ & $11058(52.6)$ & $<0.0001$ \\
\hline Fall $>2 m^{*}$ & $191(10.7)$ & $1335(11.8)$ & $883(11.1)$ & 2409 (11.5) & 0.222 \\
\hline Fall $<2 \mathrm{~m}$ & $364(20.5)$ & $1130(10.0)$ & $754(9.5)$ & $2248(10.7)$ & $<0.0001$ \\
\hline Shooting/stabbing & $50(2.8)$ & $1638(14.5)$ & $689(8.7)$ & $2377(11.3)$ & $<0.0001$ \\
\hline Blast/blow(s) & $170(9.6)$ & $1091(9.6)$ & $1066(13.4)$ & $2327(11.1)$ & $<0.0001$ \\
\hline Other & $71(4.0)$ & $316(2.8)$ & $227(2.9)$ & $614(2.9)$ & 0.018 \\
\hline \multicolumn{6}{|c|}{ Injury Severity Score (ISS) } \\
\hline Median (IQR) & $10(9-20)$ & $14(9-25)$ & $16(9-25)$ & $14(9-25)$ & $<0.0001$ \\
\hline \multicolumn{6}{|c|}{ Abbreviated Injury Scale (AIS) —-median (IQR) for highest AIS in anatomical regions } \\
\hline Head $^{*}$ & $4(3-5)$ & $4(3-5)$ & $4(3-5)$ & $4(3-5)$ & 0.126 \\
\hline Face & $2(1-2)$ & $2(1-2)$ & $2(1-2)$ & $2(1-2)$ & $<0.0001$ \\
\hline Thorax* & $3(3-4)$ & $3(3-4)$ & $3(3-4)$ & $3(3-4)$ & 0.069 \\
\hline Abdomen & $3(2-4)$ & $3(2-4)$ & $3(2-3)$ & $3(2-4)$ & 0.017 \\
\hline Spine* & $2(2-3)$ & $2(2-3)$ & $2(2-3)$ & $2(2-3)$ & 0.801 \\
\hline Pelvic & $2(2-2)$ & $2(2-4)$ & $2(2-4)$ & $2(2-4)$ & 0.0003 \\
\hline Limb & $3(2-3)$ & $3(2-3)$ & $3(2-3)$ & $3(2-3)$ & $<0.0001$ \\
\hline Other* & $1(1-2)$ & $1(1-2)$ & $1(1-2)$ & $1(1-2)$ & 0.825 \\
\hline
\end{tabular}

${ }^{*}$ No statistically significant difference.

MTC, major trauma centre; RTC, road traffic collision.

favour children's MTCs although this did not meet statistical significance. These data should be interpreted with caution due the variation in case numbers attending each MTC types (children's MTC $\mathrm{n}=17$, mixed MTC $\mathrm{n}=9153$ and adult MTC $\mathrm{n}=7507)$.

Some young adolescents (10-15.99 years) and some old adolescents (16-24.99 years) presented to non-designated centres (in accordance to English hospital set up of adult and children's MTCs), with 430 patients under 16 years of age treated in adult MTCs $(9.9 \%$ of all aged $<16$ years) and 17 patients over 16 years attending a children's MTC $(0.1 \%$ of all aged 16-24.99 years). We hypothesis that this variation in protocol is multi-factorial, including errors in estimating the age in the prehospital setting. In some instances, including in cases of extremis, prehospital teams may divert from protocol to the nearest unit for life saving interventions. In those under 16 years deemed too unstable for safe transfer the prehospital team may attend the nearest children's TU (which may also be an adult MTC).

Existing literature describing adolescent mortality secondary to trauma is predominantly from the USA, and outcomes appear contradictory across studies. A 2016 study which accessed the US National Trauma Data Bank described higher adjusted odds of mortality in adult MTCs (OR, 4.19; 95\% CI 1.30 to 13.51) and mixed MTCs (OR, 6.68; 95\% CI 2.03 to 21.99) compared with children's MTCs. ${ }^{4}$ Other studies have reported no difference

Table 3 Odds of mortality by MTC centre type for adolescent trauma patients (aged 10-24.99 years)

\begin{tabular}{|c|c|c|c|c|}
\hline \multirow[b]{2}{*}{ Centre of treatment } & \multicolumn{2}{|l|}{ Crude } & \multicolumn{2}{|l|}{ Adjusted } \\
\hline & OR $(95 \% \mathrm{Cl})$ & $P$ value & OR $(95 \% \mathrm{Cl})$ & $P$ value \\
\hline \multicolumn{5}{|c|}{ Odds of mortality for those aged $10-24.99$ years } \\
\hline Children's MTC (reference) & 1 & & 1 & \\
\hline Adult MTC & 2.02 (1.47 to 2.77 & $<0.0001$ & 2.41 (1.31 to 1.43$)$ & 0.005 \\
\hline Mixed MTC & 1.82 (1.33 to 2.49$)$ & $<0.0001$ & 1.85 (1.03 to 3.35$)$ & 0.041 \\
\hline \multicolumn{5}{|c|}{ Odds of mortality for those aged $14-17.99$ years } \\
\hline Children's MTC (reference) & 1 & & 1 & \\
\hline Adult's MTC & 1.73 (1.05 to 2.86$)$ & 0.032 & 2.77 (1.11 to 6.94$)$ & 0.030 \\
\hline Mixed MTC & 1.52 (0.95 to 2.44$)$ & 0.083 & 1.92 (0.84 to 4.42$)$ & 0.121 \\
\hline \multicolumn{5}{|c|}{ Odds of mortality for those aged $10-15.99$ years } \\
\hline Children's MTC (reference) & 1 & & 1 & \\
\hline Adult's MTC & 1.52 (0.83 to 2.76$)$ & 0.175 & 2.87 (0.79 to 10.38$)$ & 0.108 \\
\hline Mixed MTC & 1.65 (1.13 to 2.41$)$ & 0.01 & $2.22(1.1$ to 4.51$)$ & 0.027 \\
\hline \multicolumn{5}{|c|}{ Odds of mortality for those aged $16-24.99$ years } \\
\hline Children's MTC (reference) & 1 & & 1 & \\
\hline Adult's MTC & 0.24 (0.07 to 0.86$)$ & 0.03 & $3.12(0.21$ to 47.5$)$ & 0.21 \\
\hline Mixed MTC & $0.22(0.06$ to 0.79$)$ & 0.02 & $2.36(0.16$ to 35.8$)$ & 0.16 \\
\hline
\end{tabular}

Subgroup analysis of those aged 14-17.99 years, 10-15.99 years and 16-24.99 years also displayed (crude and adjusted OR presented). MTC, major trauma centre. 
Table 4 Number of patients attending each MTC type for age ranges $10-15.99$ and $16-24.99, \mathrm{n}(\%)$

\begin{tabular}{lcccc}
\hline Age group & Children's MTC & Mixed MTC & \multicolumn{1}{c}{ Adult MTC } & \multicolumn{1}{l}{ Total } \\
\hline $10-15.99$ & $1760(99.0 \%)$ & $2166(19.1 \%)$ & $430(5.4 \%)$ & $4356(20.7 \%)$ \\
$16-24.99$ & $17(1.0 \%)$ & $9153(80.9 \%)$ & $7507(94.6 \%)$ & $16677(79.3 \%)$ \\
Total & 1777 & 11319 & 7937 & 21033 \\
\hline
\end{tabular}

MTC, major trauma centre.

in mortality between centres for either penetrating or blunt trauma, ${ }^{13}$ lower crude mortality (but not adjusted mortality) in a children's trauma centre compared with an adult trauma centre, ${ }^{17}$ and no difference in mortality by centre but a reduction in imaging, invasive procedures, and ICU LoS, and improved discharge to home in children's trauma centres. ${ }^{18}$ One further study of patients with penetrating injuries (predominantly stabbing and gunshot wounds) performed a subgroup analysis on adolescents (defined as $>12$ years), finding no difference in mortality. This same study reported those $<5$ years old may have superior functional outcomes when treated at a paediatric trauma centre. ${ }^{12}$

Adolescents (10-24.99 years) who presented to a children's MTC had a lower ISS compared with adult and mixed MTCs. Previous trauma studies report a correlation between an increasing ISS and higher mortality. ${ }^{19}$ In acknowledgement of this, we undertook statistical adjustments to account for the variation in ISS and also performed a subgroup analysis for those with major trauma (ISS >15). In both, the trend for a lower mortality rate in the children's MTC remained, supporting the view that children's MTCs manage adolescent trauma with good outcomes despite seeing a lower volume of cases. This is in contrast to evidence suggesting improved trauma outcomes in institutions with higher case volumes. ${ }^{20} 21$

Between groups, there were statistically significant differences in baseline physiological observations. However, there was no clinical significance to these differences, and this finding likely represents the slight variations in normal values that one would expect over the age range of this cohort.

There was some variation in practice between trauma centres. Those that attended a children's MTC were less likely to have a consultant present. We hypothesise that this variation may be a consequence of differing patterns of conveyance to children's MTCs rather than system configuration. Up to a third of major paediatric trauma cases are more likely to 'self-present' via parental transport rather than via ambulance, removing the prealert trauma call. ${ }^{22}$ Regarding imaging, trauma cases were less

\begin{tabular}{|c|c|c|c|c|}
\hline & \multicolumn{2}{|l|}{ Crude } & \multicolumn{2}{|l|}{ Adjusted } \\
\hline & OR $(95 \% \mathrm{Cl})$ & $P$ value & OR $(95 \% \mathrm{Cl})$ & $P$ value \\
\hline \multicolumn{5}{|c|}{ Odds of mortality for those with an ISS >15 (10-24.99 years) } \\
\hline Children's MTC & 1 & & 1 & \\
\hline Mixed MTC & 1.51 (1.10 to 2.08$)$ & 0.011 & 1.80 (1.04 to 3.09$)$ & 0.035 \\
\hline Adult MTC & 1.61 (1.17 to 2.23 ) & 0.004 & $2.31(1.33$ to 4.00$)$ & 0.003 \\
\hline \multicolumn{5}{|c|}{ Odds of mortality subanalysis for ISS $>15$ in age group $14-17.99$ years } \\
\hline Children's MTC & 1 & & 1 & \\
\hline Mixed MTC & 1.56 (0.88 to 2.78$)$ & 0.132 & $2.01(0.92$ to 4.37$)$ & 0.08 \\
\hline Adult MTC & $2.73(1.23$ to 6.07$)$ & 0.014 & 2.99 (1.31 to 6.82$)$ & 0.009 \\
\hline
\end{tabular}

likely to have a CT if they presented to a children's MTC, and if they had a CT, it took longer to perform when compared with other MTC types. This reduction in imaging is in keeping with other studies, with an assumption that the difference is a result of adherence to adult imaging principles. ${ }^{811}$ Studies have noted no difference in mortality when targeted imaging is undertaken when compared with whole body CT. ${ }^{23}$ One would assume that these secondary outcomes would potentially increase mortality in children's MTCs and are unlikely to explain the variation in mortality outcomes that we noted.

There were some limitations to this study. TARN is the national trauma registry for the UK, with any analysis limited to prespecified fields. Adolescent trauma cases that do not meet TARN inclusion criteria but may have significant injuries were not captured for this analysis, potentially creating a skewed data set. The decision to exclude TUs and transfers from a TU to an MTC was taken pragmatically as the authors felt this question would be more appropriately explored separately, and because the accuracy and completeness of data may be lower than that submitted from MTCs. Thus a considerable proportion of trauma cases were not included, and although it is assumed that MTCs would have better outcomes, it is not inconceivable that TUs may have the lowest mortality. There was considerable variation in the number of cases that attended each centre type, with the lowest volume in the children's MTCs. Although this variation was accounted for in the analysis it may have impacted on the results. The chosen age range of 10-24 years is consistent with the current adolescent literature, though comparisons of trauma between a 10 -year-old and a 24 -year-old may be challenging given variations in mechanism of injury and physiology. To address this, we undertook adjusted and subgroup analysis with a smaller age range (14-17.99 years) but there is the potential that other variables have not been considered that may have impacted our findings.

Further research is required in this field to identify the factors that may have influenced these results. These should be explored qualitatively through engagement with clinicians and adolescent representatives, and quantitatively with richer interrogation of existing data on the contribution of factors such as type of injury and resulting interventions. There may also be benefit in a larger prospective study, in which all adolescent trauma cases attending EDs (not just MTCs) are collated to facilitate more comprehensive analysis.

In summary we have demonstrated that in England, children's MTCs have lower 30-day mortality rates for adolescent trauma than adult or mixed MTCs, and that this association remains following adjustment for potential confounding variables including severity of injury. Further research is required to understand factors influencing disparities in trauma-related mortality among adolescents who are cared for in non-children's MTCs. We hope that the results of this study will play a part to highlight where adolescent trauma patients are best managed, inspire further work in this field and therefore impact on future health policy for this frequently overlooked demographic group.

Twitter Damian Roland @damian_roland and Mark D Lyttle @mdlyttle

Contributors SM conceived the study. SM and OB undertook the data collection. OB performed the statistical analysis of the data. JE, HM, RB and SM interpreted the data and drafted the manuscript. All authors contributed substantially the study design and revision of the manuscript with supervision from DR, MDL and SM. All authors have approved the manuscript and agree to be accountable for the work.

Funding The authors have not declared a specific grant for this research from any funding agency in the public, commercial or not-for-profit sectors. 
Competing interests DR is a member of TARNLet (the Paediatric Working Group on behalf of TARN). OB is employed as a Medical Statistician for TARN.

Patient and public involvement Patients and/or the public were not involved in the design, or conduct, or reporting, or dissemination plans of this research.

Patient consent for publication Not required.

Ethics approval TARN has ethical approval (PIAG section 60) for research on the anonymised data that are stored securely on the University of Manchester server.

Provenance and peer review Not commissioned; externally peer reviewed.

Data availability statement Data are available upon reasonable request. Data are available upon reasonable request to the corresponding author.

\section{ORCID iDs}

Jordan Evans http://orcid.org/0000-0002-8873-7343

Damian Roland http://orcid.org/0000-0001-9334-5144

Stephen Mullen http://orcid.org/0000-0001-7115-980X

\section{REFERENCES}

1 Trauma Care UK - Home. Available: www.traumacare.org.uk [Accessed 27 Jan 2020].

2 World Health Organization. Violence and injury prevention. Available: https://www. who.int/violence_injury_prevention/key_facts/en [Accessed 20 Jun 2020].

3 Roberts Z, Collins J-A, James D, et al. Epidemiology of adolescent trauma in England: a review of TARN data 2008-2017. Emerg Med J 2020;37:25-30.

4 World Health Organization. Recognizing adolescence, 2014. Available: http://apps. who.int/adolescent/second-decade/section2/page1/recognizing-adolescence.html

5 Unicef. Available: https://unicef.org/adolescence/

6 Sawyer SM, Azzopardi PS, Wickremarathne D, et al. The age of adolescence. Lancet Child Adolesc Health 2018;2:223-8.

7 Silver K. Adolescence now lasts from 10 to 24. Available: https://www.bbc.co.uk news/health-42732442

8 Centre for Disease Control and Prevention. Preventing youth violence. Available: https://www.cdc.gov/violenceprevention/youthviolence/fastfact.html

9 Royal College of radiology. Available: https://www.rcr.ac.uk/publication/paediatrictrauma-protocols [Accessed 04 Mar 2020].
10 Matsushima K, Kulaylat AN, Won EJ, et al. Variation in the management of adolescent patients with blunt abdominal solid organ injury between adult versus pediatric trauma centers: an analysis of a statewide trauma database. J Surg Res 2013:183:808-13

11 Webman RB, Carter EA, Mittal S, et al. Association between trauma center type and mortality among injured adolescent patients. JAMA Pediatr 2016;170:780-6.

12 Miyata S, Cho J, Lebedevskiy 0, et al. Trauma experts versus pediatric experts: comparison of outcomes in pediatric penetrating injuries. J Surg Res 2017;208:173-9.

13 Walther AE, Pritts TA, Falcone RA, et al. Teen trauma without the drama: outcomes of adolescents treated at Ohio adult versus pediatric trauma centers. J Trauma Acute Care Surg 2014;77:109-16.

14 The Trauma Audit \& Research Network. Available: www.tarn.ac.uk [Accessed Jan 2020].

15 Association for the Advancement of Automotive Medicine. Abbreviated injury scale (C) 2005 update 2008. Chicago, Illinois, 2016.

16 Cousijn J, Luijten M, Feldstein Ewing SW. Adolescent resilience to addiction: a social plasticity hypothesis. Lancet Child Adolesc Health 2018;2:69-78.

17 Matsushima K, Schaefer EW, Won EJ, et al. Injured adolescents, not just large children: difference in care and outcome between adult and pediatric trauma centers. Am Surg 2013;79:267-73

18 Walther AE, Falcone RA, Pritts TA, et al. Pediatric and adult trauma centers differ in evaluation, treatment, and outcomes for severely injured adolescents. J Pediatr Surg 2016:51:1346-50.

19 Brohi K, Singh J, Heron M, et al. Acute traumatic coagulopathy. J Trauma 2003:54:1127-30.

20 Nathens ABet al. Relationship between trauma center volume and outcomes. JAMA 2001:285:1164-71.

21 Demetriades D, Martin M, Salim A, et al. The effect of trauma center designation and trauma volume on outcome in specific severe injuries. Ann Surg 2005;242:512-9.

22 The Trauma Audit \& Research Network. Severe injury in children: January 2017-December 2018. Available: https://cld.bz/hGD01ae [Accessed Jul 2020].

23 Meltzer JA, Stone ME, Reddy SH, et al. Association of whole-body computed tomography with mortality risk in children with blunt trauma. JAMA Pediatr 2018;172:542-9. 